\title{
1 From Value to Saliency: Neural Computations of Subjective Value under Uncertainty in PTSD
}

2 Authors: Ruonan Jia ${ }^{1,2}$, Lital Ruderman ${ }^{2}$, Charles Gordon ${ }^{3,4}$, Daniel Ehrlich ${ }^{1}$, Mark Horvath ${ }^{3,4}$, Serena

3 Mirchandani ${ }^{3,4}$, Clara DeFontes ${ }^{3,4}$, Steven Southwick ${ }^{3,4}$, John H. Krystal ${ }^{3,4,5,6}$, Ilan Harpaz-Rotem ${ }^{3,4, *}$, Ifat

$4 \operatorname{Levy}^{1,2,3,5,6, *}$

1. Interdepartmental Neuroscience Program, Yale University; 2. Department of Comparative Medicine,

* These authors contributed equally.

11 Corresponding authors: R.J.ruonan.jia@yale.edu; I.L. ifat.levy@yale.edu

\section{Abstract}

14 Military personnel engaged in combat are vulnerable to Posttraumatic Stress Disorder (PTSD), following

15 traumatic experiences in the battlefield. Prior research has mostly employed fear-related paradigms to unravel neural underpinnings of fear dysregulation in individuals with PTSD. The ability to acquire and update fear responses depends critically on the individual's ability to cope with uncertainty, yet the role of individual uncertainty attitudes in the development of trauma-related psychopathology has hardly been examined. Here, we investigated the association between PTSD-related alterations and the subjective 
driven by numbing symptoms. Moreover, the neural encoding of the subjective value of those uncertain options was markedly different in the brains of veterans diagnosed with PTSD, compared to veterans who

27

experienced trauma but did not develop PTSD. Most notably, veterans with PTSD exhibited enhanced representations of the saliency of rewards and punishments in the neural valuation system, especially in ventral striatum, compared with trauma-exposed controls. Our results point to a link between the function of the valuation system under uncertainty and the development and maintenance of PTSD symptoms, and stress the significance of studying reward processes in PTSD.

\section{Introduction}

Following a life-threatening experience, some individuals develop Posttraumatic Stress Disorder (PTSD) symptoms, which can be emotionally, socially and vocationally disabling. These symptoms include reexperiencing the traumatic event, avoidance of trauma reminders, and exaggerated arousal and reactivity, as well as emotional numbing (losing interest in significant activities, having difficulty experiencing happiness or love, and feeling distant from others (1)). While medications and psychotherapy help some individuals, many people with PTSD remain symptomatic following treatment (2). A better understanding of the neural basis of PTSD is crucial, as it can inform new approaches to individualized treatment.

Soldiers in combat face highly uncertain life-threatening events, which are uncontrollable (3), and that may result in serious injury to themselves or death of teammates. An individual's attitude towards uncertainty and their capacity to handle uncertainty may therefore affect one's ability to cope with potentially traumatic events. The notion of uncertainty was incorporated in studies of fear-learning attempting to unravel the behavioral and neural mechanisms of PTSD (4,5). Participants in these studies encountered probabilistic deliveries of adverse outcomes (e.g. electric shocks), and their ability to predict these outcomes was measured (e.g. by their skin conductance responses). In a separate line of work, using a behavioral economic framework, our group showed increased aversion to ambiguity (an uncertain situation where outcome probabilities are not known) in combat veterans with PTSD, compared to trauma-exposed veterans without 
PTSD, when choosing between potential monetary losses (6). This aversion to uncertainty demonstrated in

51 situations unrelated to the trauma, may also contribute to the exaggerated behavior in fear conditioning

52 paradigms, and to the development and maintenance of PTSD symptoms. Further understanding of this

53 aversion to uncertainty could provide evidence for targeting uncertainty in behavioral interventions, to

54 improve the daily decision-making and well-being of individuals with PTSD.

55 One possibility is that the increased aversion to uncertainty reflects alterations in the neural computations of subjective value in the brains of individuals who developed PTSD following trauma exposure. In the

57 general population, a network of brain regions was implicated in valuation and decision making, including the ventromedial prefrontal cortex (vmPFC), anterior cingulate cortex (ACC), posterior cingulate cortex (PCC), dorsolateral prefrontal cortex (dlPFC), ventral striatum, amygdala, and thalamus $(7,8)$. Extensive evidence suggests that the subjective value of rewards is encoded in this network (9-11), and there is also some (12-15), although less conclusive (16-20), evidence, for encoding of subjective value of punishments in the same areas. Although there is some evidence for changes in the neural processing of monetary outcomes in individuals with PTSD $(21,22)$, we do not know how uncertain decision values are encoded in the brains of these individuals. Moreover, as far as we know, the neural encoding of ambiguous losses (as opposed to gains) has not been investigated even in the general population.

66 Here we combined a simple economic task with functional MRI and computational modeling to examine

67 the neural encoding of subjective value under risk and ambiguity, and the alterations in this encoding in 68 individuals exposed to trauma. We compared combat veterans with PTSD to those who did not develop PTSD symptoms (trauma-exposed controls), and were thus able to investigate both the psychopathology of PTSD and the resilience to PTSD. We find that veterans with PTSD encode the subjective values of

71 uncertain monetary gains and losses in a U-shape manner, with increased activation for both increased gains

72 and increased losses (compatible with saliency encoding). Conversely, trauma-exposed controls encode the

73 same type of subjective values monotonically, with increased activation for increased gains, and decreased

74 activation for increased losses (compatible with value encoding). Our results suggest that this shift from 
75 value-encoding to saliency-encoding, especially of ambiguous monetary losses, could be a neural marker

76 for PTSD symptom severity.

77

78 Results

79 In an fMRI experiment, combat veterans with current PTSD diagnosis and those who never developed

80 PTSD completed a gambling task under four decision conditions on two separate days. Participants chose

81 between a sure monetary outcome (either gaining or losing \$5) and an uncertain outcome (either risky or

82 ambiguous gain or loss) (Fig 1B). Participants made decisions about gains and losses in separate blocks in

83 two scanning sessions (Fig 1A). We estimated the attitudes toward risk and ambiguity of each participant

84 through a behavioral model (see Methods) and aimed to understand the influence of PTSD symptom

85 severity on both the behavioral attitudes and the neural mechanisms of valuation. 
A. Study timeline

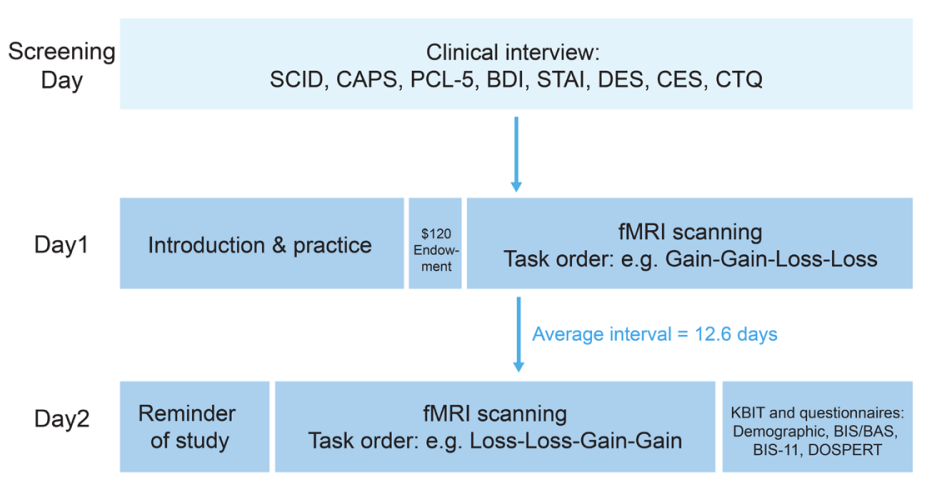

B. Task design
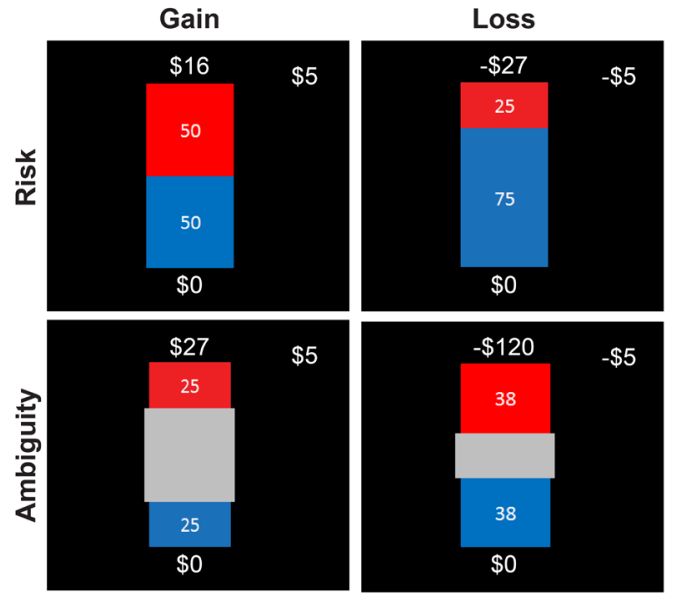

C. Lottery design

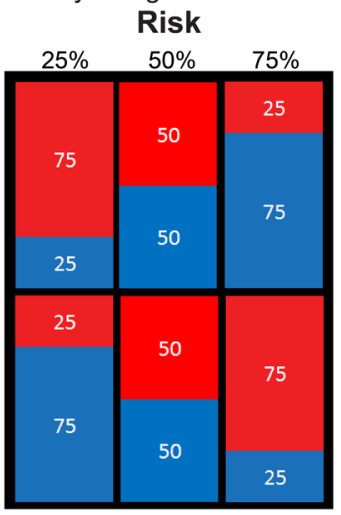

Ambiguity

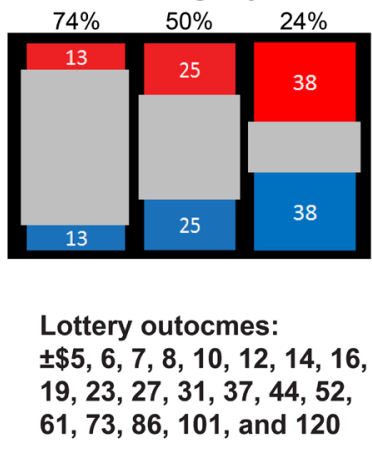

D. Trial timeline

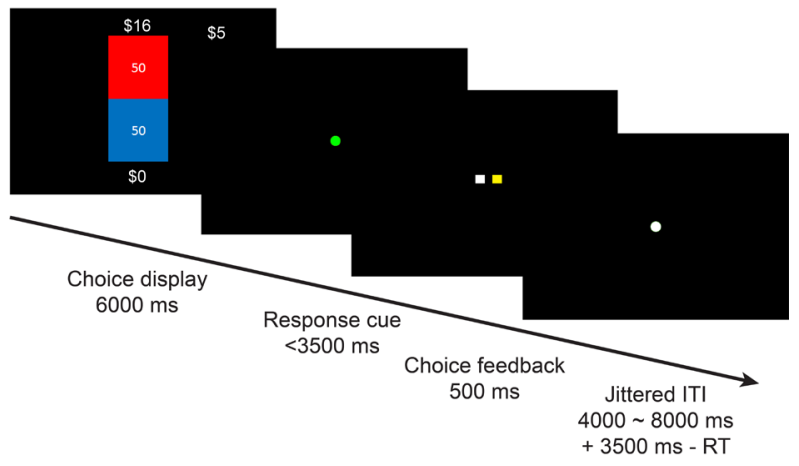

A: Timeline of the study. Participants went through a screening session and two scanning sessions on three different days. The screening session determined participants' eligibility based on PTSD diagnosis, combat exposure, and exclusion of other neurological disorders. Eligible participants were scanned on two separate days on a decision making task. Measure labels: SCID: Structured Clinical Interview for DSM-4, CAPS: Clinician Administered PTSD Scale, PCL5: , PTSD Checklist for DSM-5 , BDI: Beck Depression Inventory, STAI-1: State Anxiety, STAI-2: Trait Anxiety, DES: Dissociative Experiences Scale, CES: Combat Exposure Scale, CTQ: Childhood Trauma Questionnaire, KBIT: Kaufman Brief Intelligence Test, BIS/BAS: Behavioral Avoidance/Inhibition Scale, BIS-11: Barratt Impulsiveness Scale, DOSPERT: Domain-Specific Risk-Taking

Scale. B: Task design: participants chose between a lottery and a sure outcome under four conditions: risky gains, ambiguous gains, risky losses, and ambiguous losses. Lotteries are shown as examples. Outcome probability of the risky lottery was represented by the area of the red or blue rectangle and was fully known to 
the participant. Outcome probability of the ambiguous lottery was covered by a grey rectangle in the middle, thus was partially known to the participant. C: Levels of risk (outcome probability, $0.25,0.5$, and 0.75 ), ambiguity (grey area, $\mathbf{0 . 7 4}, \mathbf{0 . 5}$, and $\mathbf{0 . 2 4}$ ), and monetary outcomes (20 monetary gains and 20 monetary losses) of the lottery. D: On each trial, participants had 6 seconds to view the options, and made a choice following a green response cue. They had a time limit of 3.5 seconds to register the choice, after which they would immediately see a confirmation with the yellow square representing the side they chose. The lottery was not played out during the scan to avoid learning. The inter-trial-interval (ITI) was jittered among 4, 6, and 8 seconds, and the remaining time during the response window ( 3.5 seconds - response time) would be added to the ITI.

$=15.04$; control, $\mathrm{N}=34:$ Mean $=6.21, S D=9.68 ; t(34)=18.58, p<0.001)$. PTSD symptoms as captured and childhood trauma (CTQ) in the PCA. The first three components accounted for $\sim 80 \%$ of the variance

120 in those data (Fig S1A). The first component was affected by all clinical symptoms (PTSD, depression, 121 anxiety, and dissociative experiences) and might reflect a general affective factor. This component was 122 highly consistent with PTSD symptom severity (correlation with CAPS Spearman's $\rho=0.94, n=55, p<$ 123 0.001), and could be used to clearly classify PTSD diagnosis (Fig S1C). The second component was mostly 124 affected by re-experiencing, avoidance and anxious arousal clusters of CAPS, as well as the degree of 
combat exposure, potentially representing a fear learning-updating deficit or general hyperarousal. The strongly correlated with PTSD symptom severity $(\mathrm{n}=55$, Component 2: correlation with CAPS Spearman's $\rho=0.11, p=0.43$; Component 3: correlation with CAPS Spearman's $\rho=0.029, p=0.84$ ).

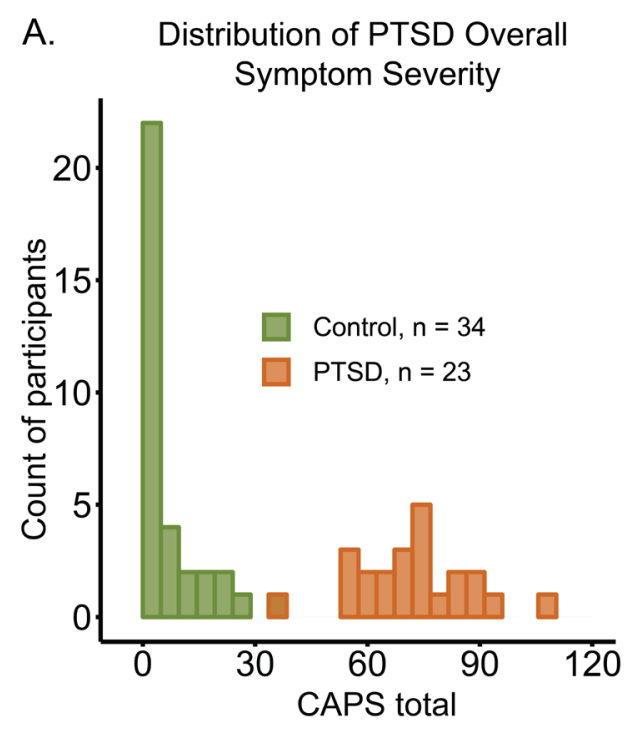

131

132
B. Correlations among Clinical Symptom Severities

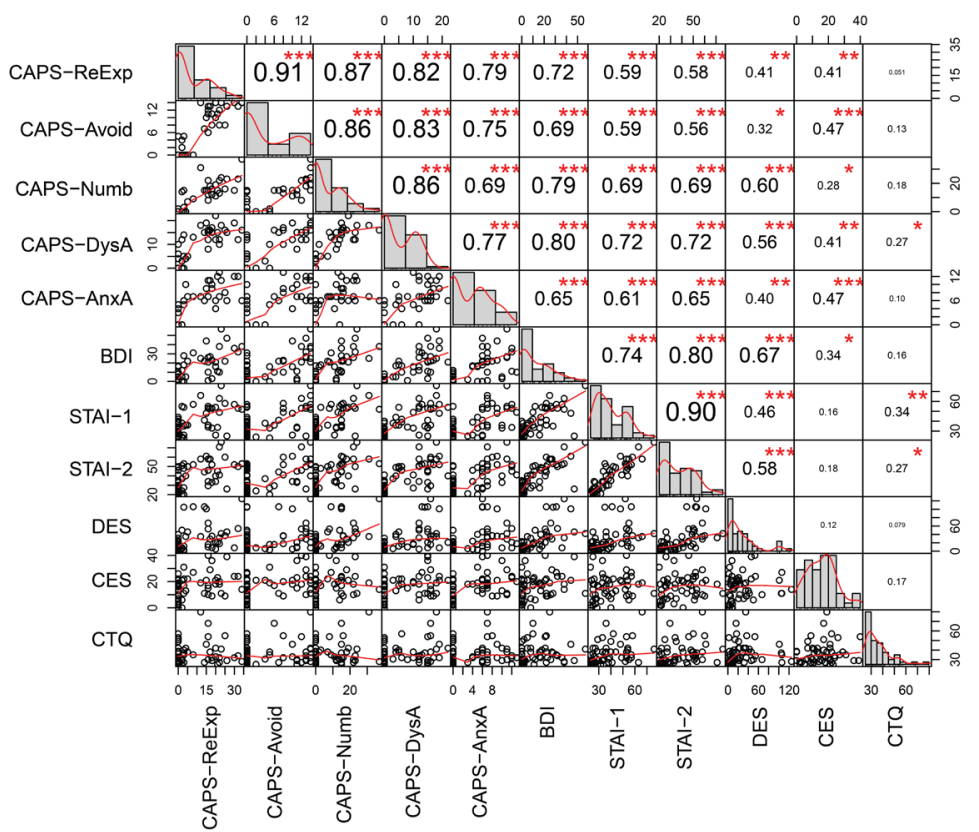

A: Distribution of CAPS total score, colored by group (combat veterans with or without PTSD diagnoses). One PTSD participant included in the analysis did not have complete CAPS data. B: PTSD, depression and anxiety symptom severities were highly correlated. Numbers in the upper right panels indicate pair-wise Pearson correlation coefficients. Significance levels: ***, $\mathbf{p}<0.001$; **, $\mathbf{p}<0.01 ; *, \mathbf{p}<0.05$. Lower left panels show pairwise scatter plots and smoothed curves using locally weighted polynomial regression. Panels in the diagonal show distributions and density curves for each measure. Labels of measures: CAPS-ReExp: re-experiencing, CAPS-Avoid: avoidance, CAPS-Numb: numbing, CAPS-DysA: dysphoric arousal, CAPS-AnxA: anxious arousal, BDI: Beck Depression Inventory, STAI-1: State Anxiety, STAI-2: Trait Anxiety, DES: Dissociative Experiences Scale, CES: Combat Exposure Scale, CTQ: Childhood Trauma Questionnaire. 


\section{PTSD symptom severity is associated with increased ambiguity aversion in the loss} domain, and increased risk aversion in the gain domain

For each participant, we estimated risk and ambiguity attitudes for gains and losses, using the combined data from both scanning sessions (see equations 1 and 2 in Methods; see Fig S2A for an example from one participant). We then investigated the associations between these attitudes and PTSD diagnosis status, as well as PTSD symptom severity. All attitudes were transformed such that negative numbers indicate aversion to risk or ambiguity, and positive numbers indicate seeking. Based on the previous behavioral finding that PTSD symptom severity was associated with higher aversion to ambiguity in losses (6), we first investigated ambiguity attitudes. At the group level, participants were not significantly averse to ambiguity in the domain of losses (Fig 3A; PTSD: Mean $=-0.25, t(23)=-1.81, p=0.11$; Control: Mean $=$ $0.003, t(33)=0.040, p=0.97$ ), and were significantly averse to ambiguity in the domain of gains (Fig $3 \mathrm{~A}$; PTSD: Mean $=-0.35, t(23)=-3.45, p<0.01$; Control: Mean $=-0.42, t(33)=-7.27, p<0.001)$. However, a two-way ANOVA of ambiguity attitude with domain as the within-subject factor and group as the between-subject factor showed a significant interaction between domain and group $(F(1,56)=4.34, p<$ $\left.0.05, \eta^{2}=0.0279\right)$. Post-hoc comparisons showed that veterans with PTSD were marginally more averse to ambiguity under losses $(p=0.081)$, but not under gains $(p=0.53)$. A dimensional analysis (Fig 3B) of this symptom-behavior relationship, regardless of PTSD diagnosis, revealed a negative correlation between ambiguity attitudes in the loss domain and CAPS total score (Spearman's $\rho$ with CAPS total score $=-0.30$, $p<0.05)$, indicating that higher symptom severity was related to higher aversion to ambiguity under losses. Since many control participants had a CAPS score of zero, we also repeated the analysis using PCL-5 scores instead of CAPS and overserved a similar effect (Fig S2B, Pearson's $r$ with PCL-5 $=-0.31, p<0.05$ ).

Next, we examined risk attitudes. Both the PTSD and control groups exhibited risk aversion in the domain of gains (PTSD: Mean = -0.54, t(23) = -13.34, $p<0.001$; Control: Mean $=-0.28, t(33)=-4.80, p<0.001)$. In the domain of losses, veterans with PTSD exhibited risk seeking (Fig 3C; PTSD: Mean $=0.34, t(23)=$ 5.43, $p<0.001)$, while combat controls exhibited marginal risk seeking (Control: Mean $=0.20, t(33)=$ $1.82, p=0.078$, FDR corrected for four comparisons). A two-way ANOVA of risk attitude with domain 
167 (gain or loss) as the within-subject factor and group as the between-subject factor revealed a significant

168 interaction between domain and group $\left(F(1,56)=6.29, p<0.05, \eta^{2}=0.0521\right)$. Post-hoc comparisons

169 showed that veterans with PTSD were more averse to risk under gains $(\mathrm{p}<0.01)$, but not under losses $(p=$

170 0.34), compared with combat controls. Examining this relationship further with a dimensional approach

171 (Fig 3D and Fig S2C), we observed a similar effect: PTSD symptom severity was negatively correlated

172 with risk attitudes in the gain domain (Spearman's $\rho$ with CAPS total $=-0.39, p<0.01$; Pearson's $r$ with

173 PCL5 $=-0.36, p<0.01)$.

174 Veterans with PTSD and combat controls did not differ in the choice noise parameter $\gamma$ (a two-way

175 ANOVA of $\gamma$ with domain (gain or loss) as the within-subject factor and group as the between-subject

176 factor: no main effect of group, $F(1,56)=1.28, p=0.262, \eta^{2}=0.0120$; no domain by group interaction,

$\left.177 F(1,56)=1.63, p=0.207, \eta^{2}=0.0136\right)$. However, model-fitting quality was in general better in the

178 control group than in the PTSD group (a two-way ANOVA of BIC with domain (gain or loss) as the

179 within-subject factor and group as the between-subject factor: a main effect of group, $F(1,56)=4.75, p<$

$1800.05, \eta^{2}=0.0587$; no domain by group interaction, $\left.F(1,56)=1.68, p=0.200, \eta^{2}=0.00788\right)$. 
Figure 3. Uncertainty attitudes and PTSD symptom severity

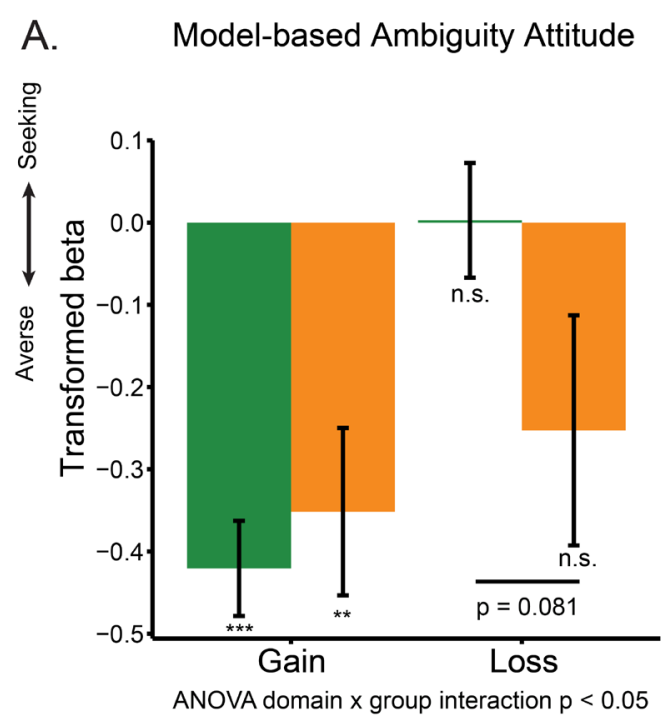

B. Ambiguity Attitude in Losses and PTSD

C.

Model-based Risk Attitude
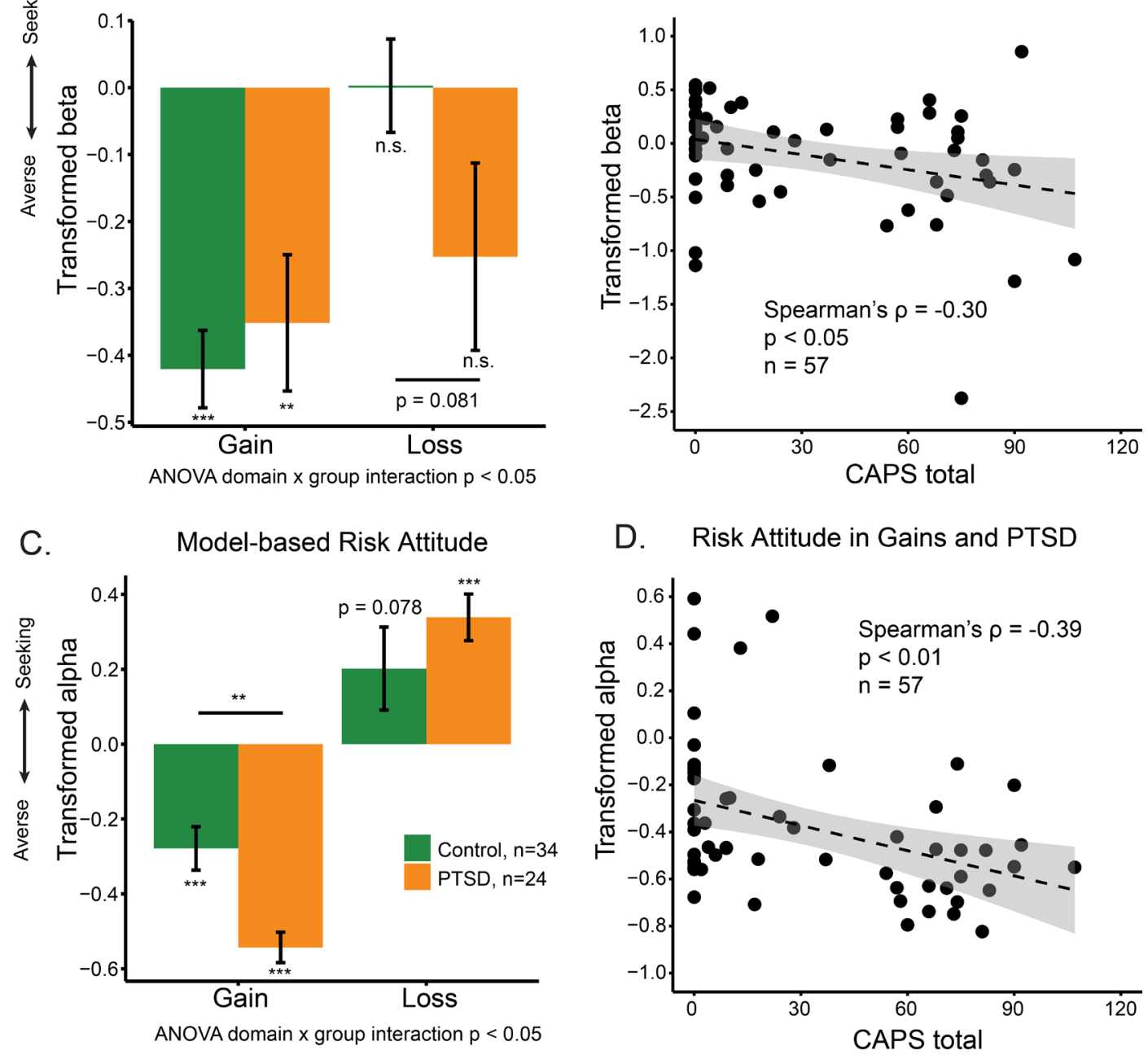

D. Risk Attitude in Gains and PTSD

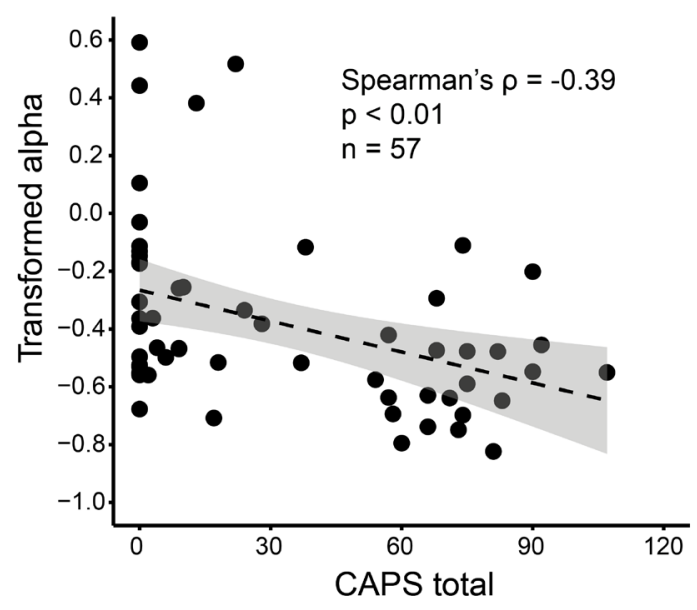

A: Group comparison of ambiguity attitudes in gains and losses between veterans with PTSD and combat controls. B: PTSD symptom severity was negatively correlated with ambiguity attitude in losses. One participant was not included in the analysis due to missing CAPS. C: Group comparison of risk attitudes in gains and losses between veterans with PTSD and combat controls. D: PTSD symptom severity was negatively correlated with risk attitude in gains. One participant was not included in the analysis due to missing CAPS. In A and C, comparisons of each group's attitudes with zero were FDR-corrected across all four comparisons in each uncertainty type. Post-hoc comparisons between groups in $\mathrm{A}$ and $\mathrm{C}$ are FDR-corrected. Significance level: *, $\mathbf{p}<0.05 ; * *, \mathbf{p}<0.01 ; * * *, \mathbf{p}<0.001$. 
To control for differences in age, income, education and intelligence, we used a linear regression model to explain uncertainty attitudes as a function of PTSD symptoms (CAPS total), while accounting for these demographic factors. Because model-fitting quality was affected by PTSD symptoms, we also included the BIC of the behavioral model as a predictor in the regression (see Supplementary Methods). For risk attitude in the gain domain, the effect of CAPS total score remained significant (multi-factor ANOVA by Generalized Linear Model: $F(1,41)=12.5, p<0.01)$. BIC was the only other significant factor $(F(1,41)=$ $17.7, p<0.001$ ). Similarly for ambiguity attitude in losses, CAPS total score (multi-factor ANOVA, $F(1$, $41)=6.05, p<0.05)$ and $\operatorname{BIC}(F(1,41)=4.86, p<0.05)$ were the only significant factors. sample (Fig S3B, D).

206 We also assessed participants' risk-taking attitudes through the Domain-Specific Risk-Taking (DOSPERT)

207 Scale self-report questionnaire, but none of the domains (Ethical, Financial, Health/Safety, Recreational, and Social) was correlated with PTSD symptoms severity measured by CAPS total. Among the other selfreport measures, CAPS total was correlated with total score of Behavioral Inhibition Scale (BIS, Spearman's $\rho=0.47, \mathrm{p}<0.001, n=57)$.

215 To investigate the neural mechanisms of the stronger aversion to uncertainty observed in veterans with 
217 our task was evaluating the subjective values of the uncertain options, we looked at the neural activity

218 during the 6-second period of options presentation on each trial (see descriptive statistics of participants

219 included in the neural analyses in Table S2). In a whole-brain analysis, we explored the relationship between

220 PTSD symptom severity and the general neural activity during this valuation process (compared to

221 baseline). Activity in a vmPFC - a central component of the valuation network - was negatively correlated

222 with CAPS total score $(p<0.001$, cluster-based corrected, Fig 4A), during the second session of the task.

223 This negative relationship was not specific to a particular condition - rather, it was consistent across all

224 four decision contexts (Fig 4B; Pearson's $r$ (risky gains) $=-0.50, r$ (ambiguous gains $)=-0.51, r$ (risky losses)

$225=-0.51, r($ ambiguous losses $)=-0.40)$. Veterans with higher overall PTSD symptom severity showed more

226 vmPFC deactivation during valuation of uncertain options. This finding is consistent with our hypothesis

227 regarding the valuation system's involvement in PTSD; next, we directly examined the neural correlates of

228 valuation in the task. 
Figure 4. Reduced vmPFC activity during valuation is related to PTSD symptom severity

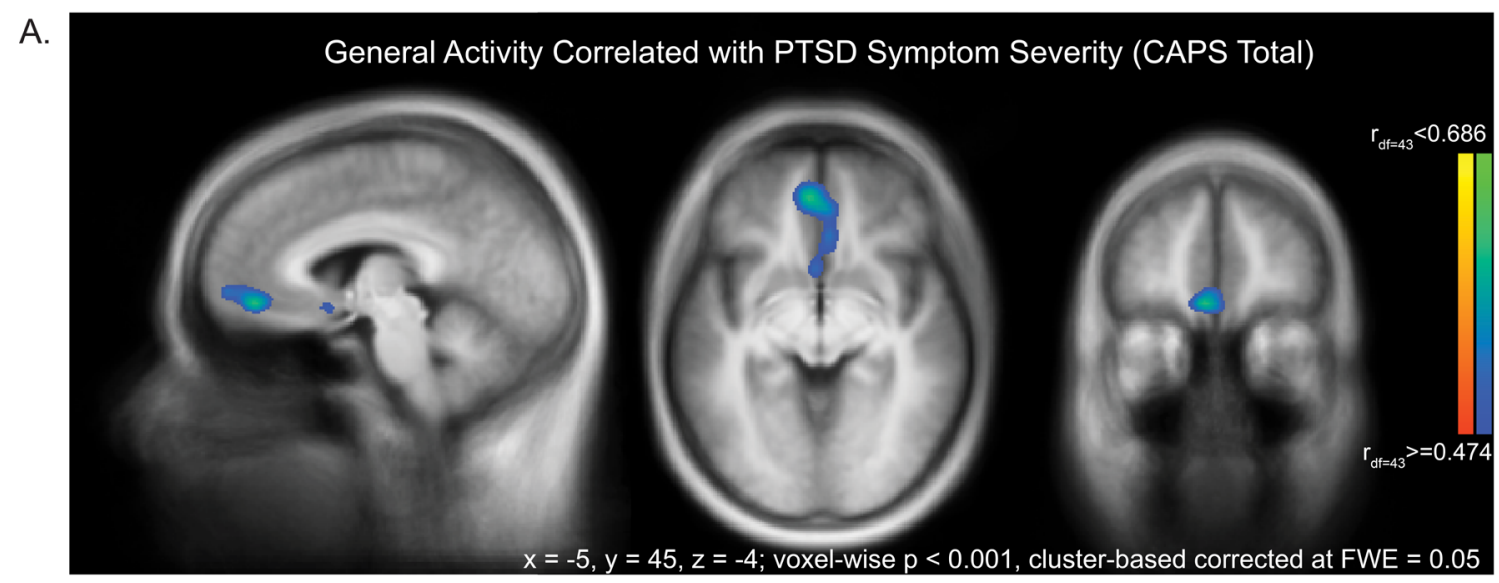

B.
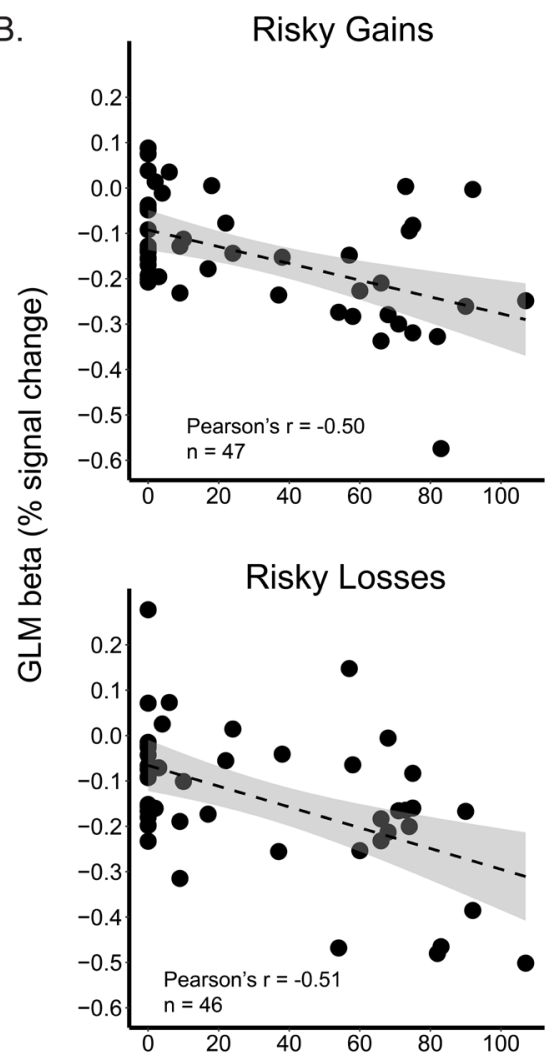

Ambiguous Gains

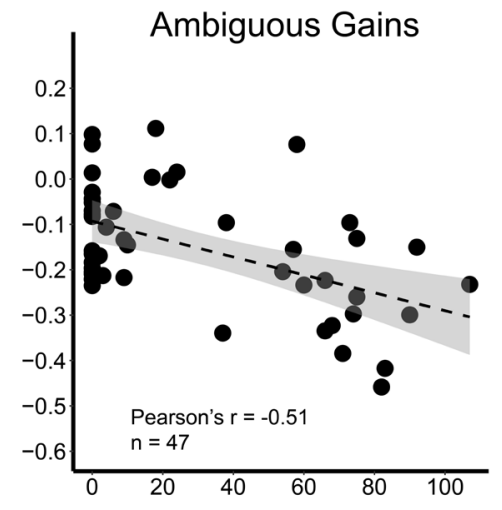

Ambiguous Losses

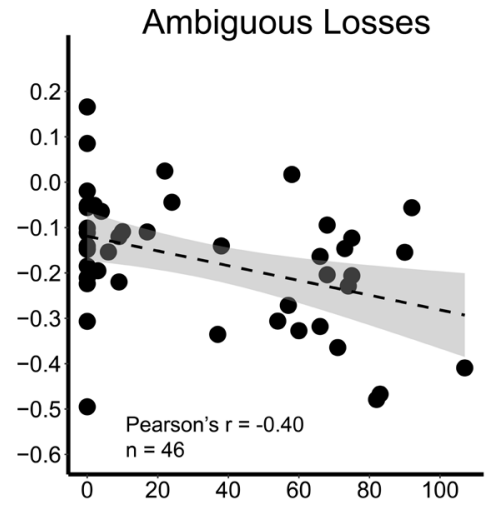

PTSD symptom severity (CAPS total)

233 A: A whole-brain analysis revealed that activity in vmPFC during valuation was negatively correlated with 234 CAPS total score, regardless of decision condition. B: Visualization of this negative correlation between general 235 activity in the vmPFC and CAPS total score in each decision condition. 

uncertain options

239 For each participant, we calculated the subjective value of the lottery presented on each trial, based on the behavioral model (see equation 1 in Methods), using the participant-specific risk and ambiguity attitudes losses in IFG was negatively correlated with PTSD symptom severity (Fig 5B; Spearman's $\rho=-0.35, p<$ $0.05, n=48)$, such that higher symptom severity was associated with more negative subjective-value signal.

252 Veterans with PTSD showed more positive subjective-value signals for risky gains in right orbitofrontal 253 cortex (OFC) in a whole-brain analysis (Fig 5C), and PTSD symptoms severity was positively correlated 254 with subjective-value signal of risky gains in this OFC region (Fig 5D; Spearman's $\rho=0.52, p<0.001, n$ $255=48$ ). For completion, we also looked at the other two conditions. Veterans with PTSD showed more 256 positive encoding of subjective value of ambiguous gains in the thalamus and right cerebellum (Fig S4), 257 and there was no group difference in the subjective-value encoding of risky losses. 
Figure 5. Neural representation of subjective value directly contrasting PTSD and control

A.

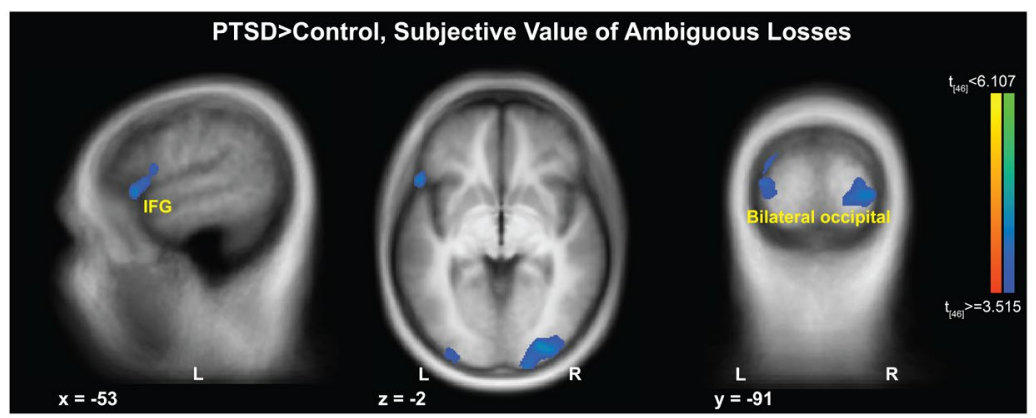

C.

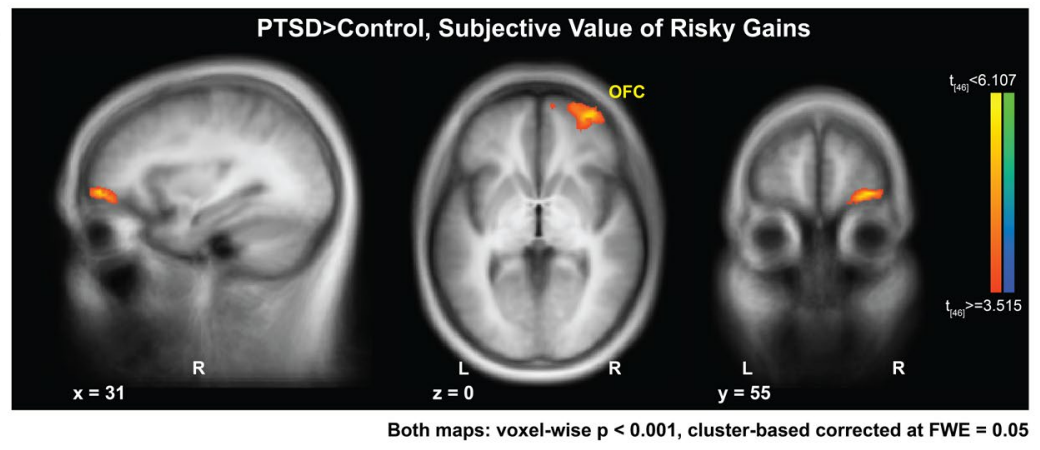

B. Ambiguous Losses, Left IFG (LOSO)

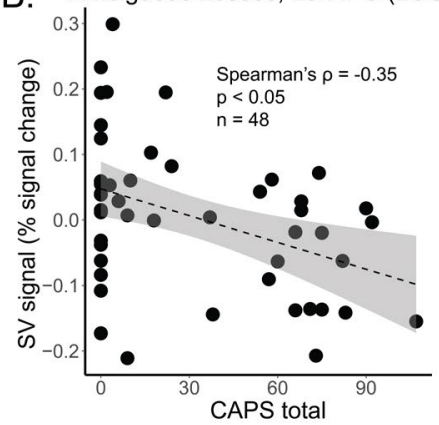

D. Risky Gains, Right OFC (LOSO)

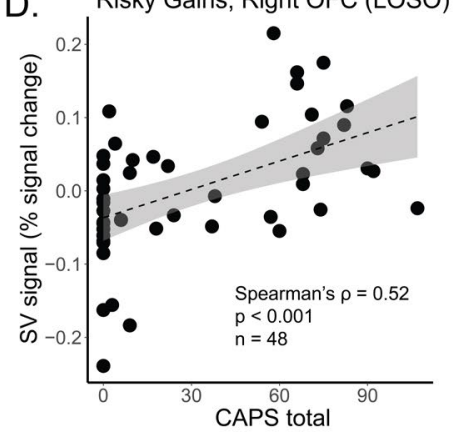

Whole-brain comparisons of neural subjective-value signals between veterans with PTSD and combat controls, under A: ambiguous losses, and C: risky gains. All maps were corrected using cluster-based method controlling family-wise error at 0.05 , when thresholded at $p<0.001$ at the voxel level. B: Neural subjective-value representation of ambiguous losses in the left IFG was negatively correlated with PTSD symptom severity. D: Neural subjective-value representation of risky gains in the right OFC was positively correlated with PTSD symptom severity. ROIs in B and D were defined by a leave-one-subject-out (LOSO) approach.

271 To further probe group and individual differences in value encoding, we examined the subjective-value 272 signals of each group in the classical value areas - the vmPFC and the ventral striatum - as defined in a 273 meta-analysis by Bartra and colleagues (14). We again focused on the conditions of ambiguous losses and 274 risky gains (Fig 6). In vmPFC, the subjective-value signal of risky gain lotteries was positively correlated 275 with PTSD symptom severity (Fig 6A, Spearman's $\rho$ with CAPS $=0.31, p<0.05$ ). In ventral striatum, 276 subjective-value signal of ambiguous loss lotteries was negatively correlated with PTSD symptom severity 
277 (Fig 6B, Spearman's $\rho$ with CAPS $=-0.35, p<0.05$ ). PTSD symptom severity was not significantly 278 associated with the subjective-value signal of ambiguous losses in vmPFC (Fig 6A, Spearman's $\rho$ with 279 CAPS $=-0.18, p=0.22$ ), or with the subjective-value signal of risky gains in ventral striatum (Fig 6B, 280 Spearman's $\rho$ with CAPS $=0.22, p=0.14$; see Fig S5 A and B for correlations with PCL5). These 281 relationships could also be revealed in the group comparison (Fig $6 \mathrm{C}$ and D). The subjective-value signal 282 of ambiguous losses was more negatively encoded in ventral striatum in veterans with PTSD compared 283 with combat controls (Fig 6D, $t=-2.77, p<0.01$ ). Conversely, the subjective-value signal of risky gains 284 was marginally more positively encoded in vmPFC in veterans with PTSD than in combat controls (Fig $2856 \mathrm{C}, t=1.97, p=0.054)$.

286 The relationships between subjective-value signals and PTSD symptom severity held after controlling for 287 age, income, education and intelligence (see Supplementary Methods). The subjective-value signal of 288 ambiguous losses in ventral striatum was affected by CAPS (multi-factor ANOVA by Generalized Linear 289 Model, $F(1,33)=6.01, p<0.05)$, and not by the four demographic factors. The subjective-value signal of 290 risky gain lotteries in vmPFC was marginally affected by CAPS (multi-factor ANOVA by Generalized 291 Linear Model: $F(1,33)=3.53, p=0.069)$, and not by the four demographic factors. 


\section{Neural Representation of Subjective Value:}

Comparing PTSD and Control

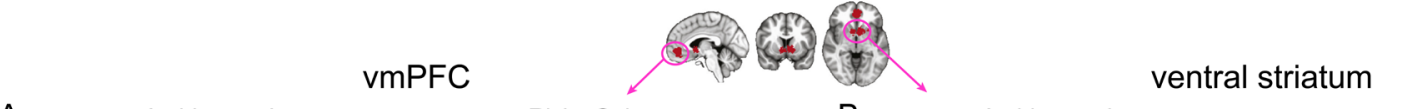

A. Ambiguous Losses

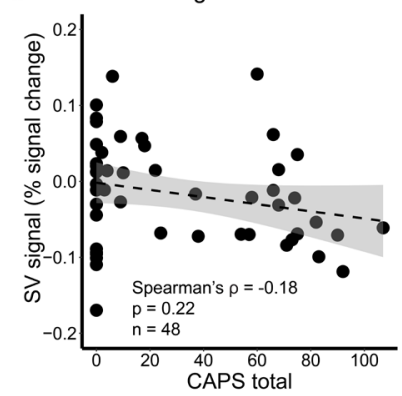

C.

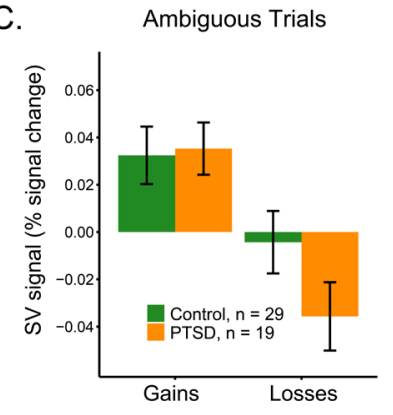

Risky Gains
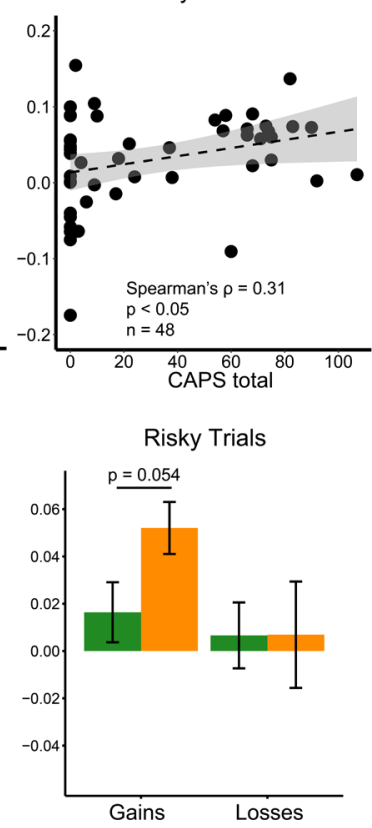

B.
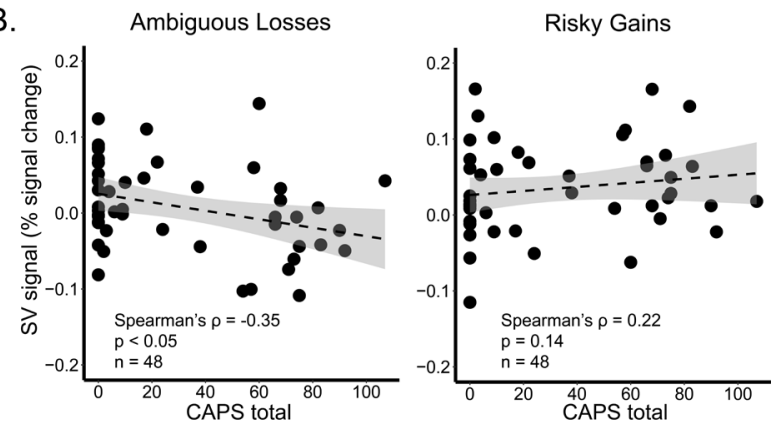

D.

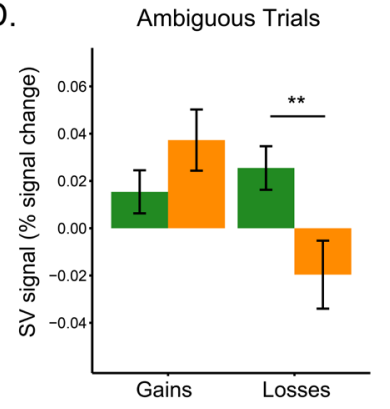

Risky Trials

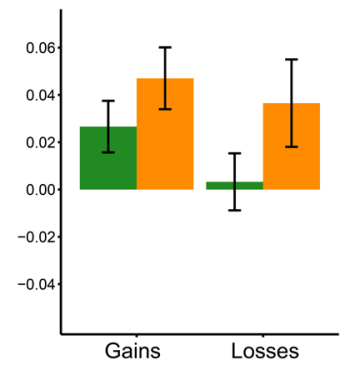

A: In vmPFC, correlations between subjective-value signals of ambiguous losses and risky gains and PTSD symptom severity (CAPS total). B: In ventral striatum, correlations between subjective-value signals of ambiguous losses and risky gains and PTSD symptom severity (CAPS total). C: In vmPFC, group comparison of neural subjective-value signals between veterans with PTSD and combat controls. D: In ventral striatum, group comparison of neural subjective-value signals between veterans with PTSD and combat controls. In panels C and D, comparisons were post-hoc FDR-corrected after ANOVA within each figure. Significance level: $*, \mathbf{p}<0.05 ; * *, \mathbf{p}<0.01 ; * * *, \mathbf{p}<0.001$. ROIs of vmPFC and ventral striatum were taken from Bartra and colleagues' meta-analysis study (14). 
307 Our results so far point to differences in the mechanisms of subjective-value encoding between veterans with PTSD and combat controls. This difference is most notable for ambiguous losses: in combat controls, ambiguous losses were encoded in a positive manner (decreased activity for increased losses) consistent with a monotonic representation of value. Conversely, in the brains of veterans with PTSD, losses were encoded negatively (increased activity for increased losses), consistent with a U-shaped saliency-encoding mechanism. This difference in representation was particularly striking in the ventral striatum (Fig 6D). To directly confirm this group difference, however, we need to examine gains and losses on the same scale.

314 To this end, we constructed a GLM with one predictor for the value of ambiguous gains and losses, and 315 another predictor for the saliency of the same gains and losses. Subjective values of the lotteries were used 316 for the value predictor, and saliency was computed as the absolute value of these subjective values (Fig 7A;

317 see Methods for fMRI GLM first-level analysis). While the ventral striatum in controls significantly 318 encoded value (one-sample t test GLM beta compared with $0, t(28)=3.4, p<0.01$ ), but not saliency $(t(28)$ $319=-0.62, p=0.54)$, the opposite pattern was observed in veterans with PTSD: activity in the same brain area 320 in the PTSD group encoded saliency $(t(18)=2.7, p<0.05)$, but not value $((t(18)=0.99, p=0.45$; all $p$ 321 values were FDR corrected for four comparisons). Furthermore, the saliency-encoding patterns were 322 significantly different between veterans with PTSD and combat controls (two-sample $\mathrm{t}$ test: $t(39.3)=-2.5$, $323 p<0.05)$.

324 Figure 7B presents a direct visualization of the shape of value- and saliency- encoding in the ventral striatum 325 in the two groups of veterans. For each participant, and within each uncertainty domain (risk or ambiguity), 326 we grouped the trials into 6 bins across losses and gains, based on the subjective values of the lotteries. The $3271^{\text {st }}$ bin corresponded to the loss lotteries with the most negative subjective values, and the $6^{\text {th }}$ bin 328 corresponded to the gain lotteries with the most positive subjective values (see Methods for details). As 329 expected, combat control veterans showed a monotonic representation of subjective value, whereas veterans 330 with PTSD showed a U-shaped representation (Fig 7B). 
Figure 7. Value and saliency encoding in the ventral striatum of PTSD and controls.

\section{Neural Representation of Value vs. Saliency: Comparing PTSD and Control}

A.
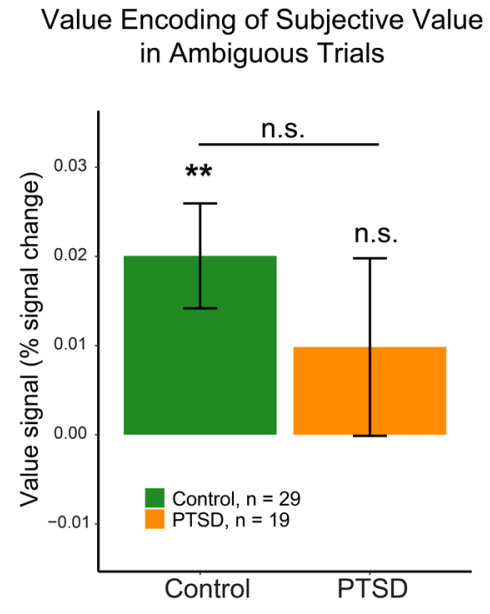

B. Value Encoding of Subjective Value in Ambiguous Trials

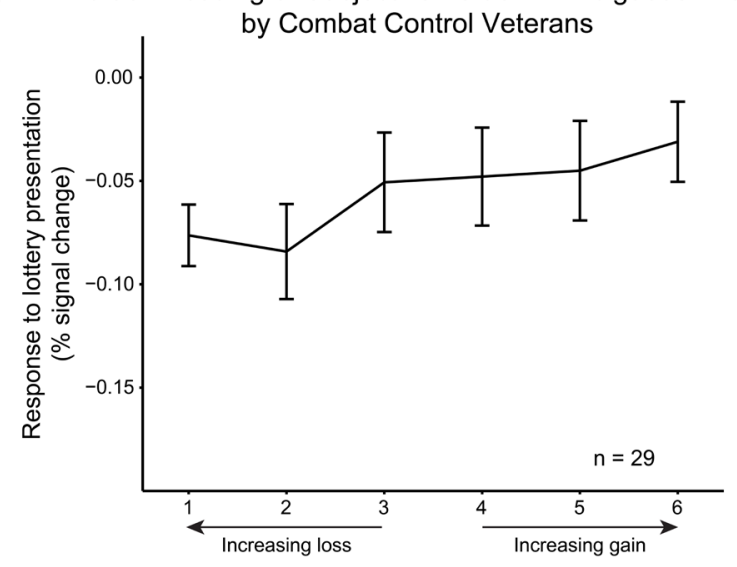

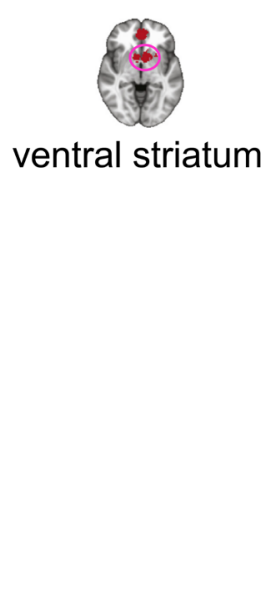
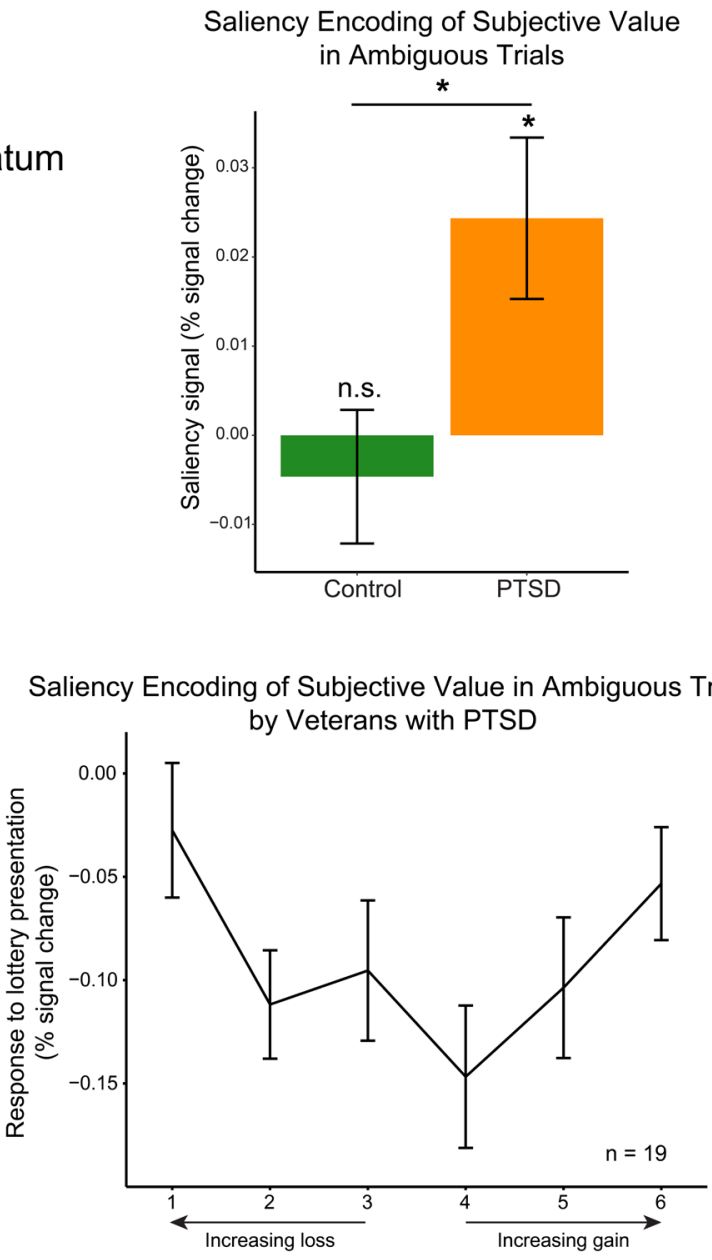

Bin of trials ordered by lottery subjective value

A: In ventral striatum, value-encoding of subjective values was observed in combat controls but not in veterans with PTSD; saliency-encoding of subjective values was observed in veterans with PTSD but not in combat controls. Comparisons with zero for both PTSD and Control group were FDR-corrected across four comparisons in the two figures. Significance level: *, $p<0.05 ; * *, p<0.01 ; * * *, p<0.001$. B: Direct visualization of neural response to trials of ambiguous lotteries with different levels of subjective values in ventral striatum. Bins were ordered monotonically based on participant-specific subjective values of the lotteries across losses and gains. Bins 1-3 were loss lotteries, and bins 4-6 were gain lotteries. Consistent with panel A, combat control veterans encoded subjective value in a monotonic value-pattern, and veterans with PTSD 


\section{Emotional numbing plays the key role in diminished vmPFC general neural activity}

356 So far, we investigated the relationship between PTSD overall symptom severity and valuation under 
influence vmPFC neural activity (standardized regression coefficient, Age: Beta $=-0.14, t=-1.13, p=0.26$; intelligence: Beta $=-0.044, t=0.33, p=0.75$ ). Variable selection using exhaustive search also indicated that including only the emotional numbing cluster out of all PTSD symptom clusters best explained the relationship between vmPFC neural activity and PTSD symptom severities (Fig S7B, BIC = 112.8; see details in Methods for fMRI GLM second-level analysis).

373 Because our participants showed high levels of comorbidity with other clinical symptoms, especially 374 depression and anxiety (Fig 2B), we also investigated how the behavioral and neural mechanisms of valuation were influenced by symptoms beyond PTSD. We examined the correlation between the first three principal components of all clinical measures (Fig S1) and the behavioral uncertainty attitudes. Principal component 1 (general affective symptom) was negatively correlated with risk attitude under gains (Pearson's $r=-0.35, p<0.01$ ) and ambiguity attitude under losses (Pearson's $r=-0.29, p<0.05$ ), consistent with the effect of the overall PTSD severity indicated by CAPS total score (Fig 3). We did not find any relationship between uncertainty attitudes and the second (fear learning-updating) or the third (trauma severity) principal components.

We also examined potential relationships between subjective-value signals and the three principal components. In vmPFC, the first component (general affective symptom) was positively correlated with encoding of subjective value of risky gains (Pearson's $r=0.30, n=47, p<0.05$ ), consistent with the effect of PTSD symptom severity (CAPS total score). The third component (trauma severity) was negatively correlated with encoding of subjective value of ambiguous losses (Pearson's $r=-0.29, n=47, p<0.05$ ), in the same direction as the correlation between subjective value of ambiguous losses and PTSD symptom S5C). 
392 In this study, we explored the neural basis of valuing uncertain monetary rewards and punishments, in veterans exposed to combat trauma with a wide range of PTSD symptoms. Behaviorally, symptom severity was associated with increased aversion to ambiguous losses, and increased aversion to risky gains. These two conditions were also the ones in which PTSD symptom severity influenced the neural representations of subjective value (Fig 7). Two main effects were observed: first, in both whole-brain and ROI analyses, veterans with PTSD showed more negative neural representation of ambiguous losses, and more positive neural representation of risky gains, than combat control veterans. Second, there was a qualitative group potential large losses), whereas in combat control veterans it encoded the lottery value. Moreover, a direct examination of the neural response to varying subjective values (Fig. 7B) suggests that the value pattern in controls was weak compared to the saliency pattern in PTSD. An intriguing possibility is that the strong neural tracking of saliency is a marker for vulnerability to PTSD, reflecting increased sensitivity to highly salient stimuli. The value signal, on the other hand, may be a marker of resiliency to PTSD. Future research, and in particular longitudinal studies that compare individuals exposed to trauma to those who never experienced trauma, are needed to explore this possibility.

\section{Using behavioral economics to identify markers of psychopathology}

410 Our results add to a growing body of research, demonstrating the utility of behavioral economics in studying

411 psychopathology (24-28). Replicating the previous behavioral study (6), we found an association between

412 higher PTSD symptom severity and greater ambiguity aversion under losses, in an independent combat

413 veteran sample. It should be noted, however, that this effect is weak, and was not significant in the group 414 comparison. A larger sample is needed to further confirm the robust effect of the relationship. We also 415 identified greater aversion to risk under gains in veterans with PTSD, likely due to a task design with 
increased range and variance of monetary outcomes that provided higher sensitivity for capturing true uncertainty attitudes. Our neural measure allowed us to also quantify individual and group differences in neural sensitivity to rewards and punishments. Previous studies have shown alterations in the neural processing of aversive outcomes in individuals with PTSD in various brain areas, including several medial and lateral prefrontal regions. Many of these studies, however, used fear and trauma-related stimuli (29).

421 Here we show that activation in the same brain areas is affected by PTSD symptoms even in an economic

422 decision task, completely unrelated to the trauma. This raises the possibility of developing diagnostic 423 methods in the domain of decision making under uncertainty, which do not require patients to recall the 424 traumatic experience. Several previous studies have also reported altered reward processing in PTSD (30), 425 including reduced expectation of uncertain monetary outcomes $(31,32)$ and decreased differentiation between monetary gains and losses in the striatum (22). Our experimental approach allowed us to estimate 427 individual uncertainty attitudes during active decision making under four unique contexts. We applied a well-established computational model to infer these behavioral individual differences from the observed choice behavior (rather than estimating them through self-reports) and used the individual differences in the analysis of the neural data. Interestingly, participants' self-reported risk-taking on the DOSPERT 431 questionnaire was not strongly correlated with their PTSD symptom severity, suggesting that our method 432 for estimating uncertainty attitudes through a behavioral task may be more sensitive for capturing subtle differences associated with clinical symptoms. An intriguing question remains to be answered is what contributes to the context-specific differences associated with PTSD symptom severity. We found higher behavioral aversion only to ambiguous losses and risky gains in PTSD, and the most striking neural 436 difference of subjective-value encoding was revealed in the context of ambiguous losses. Compared with 437 risky outcomes, of which both outcome magnitude and probability are known, ambiguous outcomes lack 438 the exact information of outcome probability. This additional level of uncertainty may be more relevant to 439 the nature of battlefield, making negative ambiguous outcomes more relatable to combat exposure. Our 440 task design separating decision contexts enabled us to pinpoint specific cognitive processes affected by 441 PTSD in combat veterans, but replication in larger samples is needed to confirm the results. 
443 By including both monetary gains and losses in the task design, we identified a shift from value-encoding

444 to saliency-encoding in the brains of individuals who developed PTSD following trauma exposure (Fig 7).

445 This shift could potentially imply an attention or arousal signal, that leads to avoidance of aversive

446 outcomes like uncertain monetary gains or losses. Several previous studies examined the neural processing

447 of value and saliency and revealed both distinct and overlapping regions for each type of encoding. Value

448 signals were found in ventral striatum, parietal cortex, OFC, rostral ACC, and saliency signals were found

449 in ventral striatum, rostral ACC, dorsal ACC, anterior insula by both univariate and multivariate analyses

$450(12,13,15-18,33,34)$. To our knowledge, our results are the first to recognize the influence of psychiatric

451 symptoms in humans on the value/saliency-encoding pattern. Interestingly, recent research in mice shows

452 a similar flip in representation, where acute stress transforms reward responses in the lateral habenula into

453 punishment responses (35). Neurons in the nucleus accumbens of rats can also flexibly shift their

454 preferences between rewards and punishments, based on the emotional environment (36), suggesting that

455 what we observe here may reflect a stress coping mechanism.

456 PTSD is highly comorbid with symptoms of depression and anxiety. Through PCA, we were able to

457 disentangle three main symptom components, and showed that the component of general affective 458 symptoms was likely the main source of influence (Fig S5). In addition, we also found that the component 459 of trauma symptoms was related to the neural representation of subjective value of ambiguous losses in 460 vmPFC, raising the possibility that trauma exposure additionally influences sensitivity to aversive monetary 461 outcomes, independent from general affective symptoms. Trauma symptoms were assessed through combat 462 exposure and childhood trauma in our sample of veterans. Future research could investigate more 463 systematically how other types of trauma exposure could additionally influence the neural processing of 464 valuation of uncertain outcomes.

465 One concern in our investigation of neural representation of value is that the range of subjective values is 466 lower in the group of veterans of PTSD because of their higher aversion to uncertainty, which could 
467 influence the sensitivity of the neural response to value differences. It should be noted, however, that our

468 main conclusion is based on a difference in the direction of correlation (negative vs. positive), rather than

469 a difference in the magnitude of slope of the correlation (Figs 6 and 7). This represents a substantial

470 difference in the shape of subjective-value encoding and would not be affected by group difference in the

471 range of subjective values.

474 Previous studies of PTSD often focused on the neural processing of fear and trauma, and identified both

475 functional and structural abnormalities in amygdala, hippocampus, and vmPFC (5,29,37-39). Other studies

476 have looked into more general cognitive processes and found blunted neural activation to monetary rewards

477 (21,22). In our study, using a more nuanced computational approach, PTSD symptoms were associated

478 with increased neural sensitivity to rewards and opposite direction of sensitivity to punishments. While the

479 sensitivity to rewards may seem at odds with the previous studies, it should be noted that in those studies

480 reward signals were defined as the difference in activation to gains and losses. A weaker contrast in

481 individuals with PTSD could stem from a weaker reward signal, but also from a stronger punishment signal,

482 consistent with a U-shaped saliency representation, as we report here, in which both highly salient positive

483 and highly salient negative outcomes elicit similar magnitude of neural activation. With this being said, in

484 the reward domain, we do find evidence of surprisingly stronger value sensitivity in PTSD (Fig 5 and 6).

485 Note that reduced activation to rewards in individuals with PTSD was previously observed in comparison

486 to controls who were not exposed to trauma (21). In our study, veterans with PTSD exhibited neural patterns

487 for potential rewards which were similar to what has been observed in the general population $(9,14)$.

488 Combat controls, who were exposed to trauma, but did not develop PTSD, were the ones who differed from

489 the general population, suggesting that they have exhibited a resiliency marker. Other important

490 methodological details may contribute to the difference in results between the studies, including a focus on

491 outcome delivery, rather than decision value, female participants, and mixed types of trauma. These 

uncertainty and combat trauma, as uncertainty is a central component of the battlefield experience. Comparing behavior and neural mechanisms in individuals who experienced combat trauma and those who did not, will help to shed light on the potential vulnerability and resiliency markers proposed here. substance abuse, to allow for a diverse representative sample of trauma exposed symptomatology. We controlled for substance abuse by conducting urine test and breathalyzer for anyone with substance abuse history or if we suspected any intoxication, and excluded those with positive results. The severity for substance abuse history in our sample was low and did not vary too much as measured by the Addiction Severity Index (ASI-alcohol: median $=0.089$, range $=[0,1.47]$; ASI-drug: median $=0$, range $=[0,0.092])$. Future research could better control for substance abuse history and medication, and potentially look into the pharmacological effect involving the dopamine and serotonin systems, which are crucial for valuebased decision making $(40,41)$.

505 Our study could not establish causal relationship between decision making under uncertainty and the 506 development of PTSD symptoms. Heightened aversion to uncertainty could possibly predispose individuals 507 to developing PTSD symptoms, and on the other hand, acquiring PTSD symptoms could result in altered 508 uncertainty attitudes. There is some evidence, however, that risk attitude is correlated with relatively stable 509 biomarkers including structural volume of right posterior parietal cortex (42), structural and functional connectivity of the amygdala (43) and genetic variations (44). These pieces of evidence might indicate that risk attitude is a personal trait, raising the possibility of its predisposing effect on the development of PTSD

512 symptoms. Less evidence exists for biomarkers of ambiguity attitude, although there is some evidence for 513 a genetic association among females (45). Further longitudinal studies comparing veterans pre- and post514 military service may disentangle the role of pre-existing uncertainty attitudes on the development of PTSD 515 from the subsequent impact of PTSD symptomatology on uncertainty attitudes. 
Variations in decision making under uncertainty, and especially under ambiguity, have also been reported

517 in other psychiatric disorders, including higher ambiguity aversion and choice inconsistency in individuals

518 with Obsessive Compulsive Disorder (25), and decreased ambiguity aversion in individuals with antisocial

519 personality disorder (26). Interestingly, a recent longitudinal study demonstrated transient increases in

520 tolerance to ambiguity before relapses in opioid users undergoing treatment (27). Overall, these efforts to

521 study psychiatric disorders using behavioral economics approaches could collectively lead to both early

522 identification of behavioral and biological risk factors for symptom development, and more effective

523 treatment.

\section{Methods}

526

\section{Participants}

52768 male veterans (ages: 23.6-74.6; mean \pm standard deviation: $39.4 \pm 11.5$ ), who had been deployed and 528 exposed to combat, were recruited through flyers and were screened by clinicians at West Haven Veterans

529 Affairs hospital. Due to the small proportion of female combat veterans (15\% of female in Army 2019 , statistics from Department of Defense), we only included male participants. PTSD sympotms and diagnoses were determined by the Structured Clinical Interview for DSM-4 (SCID) (46) and the Clinician Administered PTSD Scale (CAPS) (23). Participants either had current diagnoses of PTSD at the time of the study or were never diagnosed with PTSD (controls). We also collected the following measurements: and Childhood Trauma Questionnaire (CTQ) (52). Participants with psychosis, bipolar disorder, traumatic

537 brain injury, neurologic disorder, learning disability, and ADHD were excluded after screening. Participants 538 also completed other questionnaires including demographic information, Behavioral Avoidance/Inhibition 539 (BIS/BAS) Scales (53), the Barratt Impulsiveness Scale (BIS-11) (54), and Doman-Specific Risk-Taking 
a measure of non-verbal intelligence.

542 Participants data was excluded based on behavioral quality check (see Supplementary Methods) and 543 excessive movement in the scanner. Behavioral data of 58 participants (ages: 23.6-67.0; mean \pm standard 544 deviation: $37.3 \pm 8.9$ ) and neural results of 48 participants (ages: 23.6-67.0; mean \pm standard deviation: $54537.4 \pm 9.2$ ), were reported. The study was approved by the Yale University Human Investigating Committee and the Human Subjects Subcommittee of the VA Connecticut Healthcare System, and compliance with all

547 relevant ethical regulations was ensured throughout the study. All participants gave informed consent and were compensated with $\$ 100$ for their participation, plus a variable bonus $(\$ 0-\$ 240)$ based on choices they made in the task (see Supplementary Methods).

\section{Experimental design}

552 The study was composed of three separate visits on three different days (Fig 1A). On the first day, recruited 553 participants went through clinical interviews for screening. Eligible participants continued to two fMRI 554 sessions, on two separate days. In the scanner, participants performed a task of decision making under 555 uncertainty, which is based on a previous neuroimaging study (57) and similar to the design of a previous 556 behavioral study in combat veterans (6). They made a series of decisions between a sure monetary outcome 557 and an uncertain monetary outcome with either known (risky) or unknown (ambiguous) outcome probability, in scenarios of both gaining and losing money (Fig 1B). On each trial, participants viewed the two options side-by-side for a fixed duration of 6 seconds, and then made a choice (Fig 1D). To prevent learning, the outcome of the chosen option was not presented during the scan. At the end of the experiment, one randomly selected trial was realized for bonus payment. The scans were conducted over two days in 


\section{MRI scans}

MRI data were collected with two scanners (due to scanner upgrade) at the Yale Magnetic Resonance Research Center: Siemens 3T Trio (37 participants, 29 reported in imaging results) and 3T Prisma (31 participants, 19 reported in imaging results), using a 32-channel receiver array head coil. High resolution structural images were acquired by Magnetization-Prepared Rapid Gradient-Echo (MPRAGE) imaging (TR $=2.5 \mathrm{~s}, \mathrm{TE}=2.77 \mathrm{~ms}, \mathrm{TI}=1100 \mathrm{~ms}$, flip angle $=7^{\circ}, 176$ sagittal slices, voxel size $=1 \times 1 \times 1 \mathrm{~mm}, 256 \times$ 256 matrix in a $256 \mathrm{~mm}$ field-of- view, or FOV). Functional MRI scans were acquired while the participants were performing the choice task, using a multi-band Echo-planar Imaging (EPI) sequence $(\mathrm{TR}=1000 \mathrm{~ms}$, $\mathrm{TE}=30 \mathrm{~ms}$, flip angle $=60^{\circ}$, voxel size $=2 \times 2 \times 2 \mathrm{~mm}, 602 \mathrm{~mm}$-thick slices, in-plane resolution $=2 \times 2$ $\mathrm{mm}, \mathrm{FOV}=220 \mathrm{~mm})$.

\section{Model-based risk and ambiguity attitudes estimation}

We fitted each participant's choice data separately into a behavioral economics model that was used in previous studies $(6,9)$. The model fitting was conducted separately for gain and loss trials. The model separates the decision process into two steps: valuation and choice. In the valuation step, the subjective value (SV) of each option is modelled by equation (1),

$$
S V=\left[P-\beta\left(\frac{A}{2}\right)\right] \times V^{\alpha}
$$

where $\mathrm{P}$ is the outcome probability $(0.25,0.50$, or 0.75 for risky lotteries, 0.5 for ambiguous lotteries, and 1 for the certain option); $\mathrm{A}$ is the ambiguity level $(0.24,0.5$, or 0.74 for ambiguous lotteries; 0 for risky lotteries and the certain amount); $\mathrm{V}$ is the non-zero outcome magnitude of the lottery or the amount of money of the certain option. For choices in the loss domain, amounts are entered with a positive sign. Risk attitude was modeled by discounting the objective outcome magnitude by a participant-specific parameter, $\alpha$. In the gain domain, a participant is risk averse when $\alpha<1$, and is risk seeking when $\alpha>1$. Because we fitted the choice data in the loss domain using positive outcome magnitudes, the participant is risk averse when $\alpha>1$, and is risk seeking when $\alpha<1$. Ambiguity attitude was modeled by discounting the lottery 
590

591

592

593

594

595

596

597

598

599

600

601

602

603

604

605

606

607

608

609

610

611

612

613

probability linearly by the ambiguity level, weighted by a second participant-specific parameter, $\beta$. A participant is averse to ambiguity when $\beta>0$, and is ambiguity seeking when $\beta<0$ in the gain domain. In the loss domain, participant is averse to ambiguity when $\beta<0$, and ambiguity seeking when $\beta>0$.

The choice process is modeled by a standard soft-max function (equation 2),

$$
P_{\mathrm{V}}=\frac{1}{1+e^{\gamma\left(S V_{\mathrm{L}}-S V_{\mathrm{C}}\right)}}
$$

where $\mathrm{P}_{\mathrm{V}}$ is the probability of choosing the lottery option, $\mathrm{SV}_{\mathrm{C}}$ and $\mathrm{SV}_{\mathrm{L}}$ are the subjective values of the certain option and the lottery respectively, calculated by equation (1); $\gamma$ is a participant-specific noise parameter. We fitted each participant's choices combining data from two sessions and obtained four attitudes: risk attitudes for gains and losses, ambiguity attitudes for gains and losses. For consistency, we transformed all attitudes in the following way such that negative values indicate aversion and positive values indicate seeking: risky gains: $\alpha-1$, risky losses: $1-\alpha$, ambiguous gains: $-\beta$, ambiguous losses: $\beta$. Since participants performed the task on two separate sessions, we also fitted each session's choice data separately. These fitted parameters from separate sessions were used to calculate trial-wise subjective values of the lotteries for GLM neural analysis, because they could capture the subjective values more accurately for searching neural activity change induced by variations of subjective values.

\section{MRI data analysis}

MRI data were preprocessed in BrainVoyager (Version 20.2.0.3065). Anatomical images were normalized to the standard brain template in Talairach space for each participant. Preprocessing of functional data included motion correction, slice scan time correction (cubic spline interpolation), temporal filtering (highpass frequency-space filter with cut-off cycle of 3), spatial smoothing (Gaussian filter with 8mm full-width at half-maximum), co-registration with high-resolution standardized anatomical data, and normalization to Talairach space. Scan data with movement of over $2 \mathrm{~mm}$ in any direction were excluded from analysis. 
614 First level GLM analysis was conducted in the Neuroelf toolbox (Version 1.0, https://neuroelf.net/) through

615 MATLAB (Version R2018b). The pre-processed fMRI signal time course was first converted to percent

616 signal change within each scanning block, and activity of each voxel was modeled by GLM predictors

617 convolved with a standard double-gamma hemodynamic response function. In the first GLM, we looked at

618 the general activity during decision making, by including four binary predictors for all four decision

619 conditions: ambiguous gains, risky gains, ambiguous losses, and risky losses. Each binary predictor was

620 modeled as a box-car function, with the duration of choice display (6TR). We modeled choice response of

621 all trials by another binary predictor with the duration of 1TR at the time of button press, and missing

622 responses were not modeled. We also included nuisance predictors of 6 motion correction parameters

623 (translation and rotation in the $\mathrm{x}, \mathrm{y}$, and $\mathrm{z}$ directions) in the GLM to account for influence of head motions

624 on the neural activity. In a second GLM, we modeled the neural response to the variation of trial-wise

625 subjective value of the lottery by including the subjective value as a parametric modulator for each of the

626 four decision-condition binary predictors. Subjective value of the lottery in each trial was calculated

627 uniquely for each participant by equation (1), by taking the fitted $\alpha$ and $\beta$ for each participant under each

628 domain of either gains or losses. Because we fitted the choice data in the loss domain by inputting the

629 positive outcome value, we flipped the sign of the calculated subjective value back in the loss domain. We

630 calculated the subjective values taking $\alpha$ 's and $\beta$ 's fitted from the two sessions separately, because it would

631 make the estimate of neural response to subjective value variation more accurate. Subjective values were

632 normalized within each scanning block before GLM fitting, so that the estimated effect reflected each

633 participant's neural response to the variation of subjective value, rather than to its absolute magnitude.

634 Predictor of choice response and nuisance predictors of motion correction were included as in the first

635 GLM. In the third and fourth GLMs, we aimed to further investigate the shape of the neural representation

636 of subjective values. In both GLMs, we combined trials of gains and losses, and only separated trials by

637 uncertainty types. Thus, we included two binary predictors, ambiguous trials and risky trials, in both GLMs,

638 and modeled them as box-car functions with a duration of choice display (6TR). In the third GLM, we

639 included the subjective value itself as a parametric modulator to accompany each binary predictor, to look 
at the monotonic value-encoding of subjective values. In the fourth GLM, we included the absolute value

641 of subjective value as a parametric modulator to accompany each binary predictor, to look at the U-shaped

642 saliency-encoding of subjective values. The predictor of choice response and nuisance predictors of motion

643 correction were included as in the first GLM. In the fifth GLM to more directly visualize the subjective-

644 value encoding pattern, we made binary predictors based on the subjective value of the lottery. For each

645 participant, we first separated all trials into risky and ambiguous one. Within each uncertainty domain, we

646 then grouped loss trials into 3 bins, by comparing the subjective value of the lottery in each trial to the $1 / 3$

647 and 2/3 quantile value of the subjective values of all the loss lotteries in this uncertainty domain. Similarly,

648 we grouped gain trials into 3 bins, by comparing the subjective value of the lottery in each trial to the $1 / 3$

649 and 2/3 quantile value of the subjective values of all the gain lotteries in this uncertainty domain. We then

650 constructed a binary predictor for each bin as a box-car function with the duration of choice display (6TR).

651 Altogether this GLM included 12 predictors ( 2 uncertainty domains $\times 2$ gain/loss domain $\times 3$ bins)

652 representing the levels of subjective values. Within each uncertainty domain, there were 6 bins of trials,

653 and the $1^{\text {st }}$ bin included the loss lotteries with the most negative subjective values, and the $6^{\text {th }}$ bin included

654 the gain lotteries with the most positive subjective values. An additional predictor of response was modeled

655 as the same way as the other GLMs.

656 In the second-level analysis, random-effect group analysis was conducted to test whether the mean effect

657 of interest was significantly different from zero across participants, or significantly different between

658 groups by contrasting veterans with PTSD and combat controls. We also took a dimensional approach to

659 test whether the predictor effects were related to the severity of PTSD and other clinical symptoms. The

660 tests were conducted both in a whole-brain search and in ROIs. All whole-brain statistical maps were

661 thresholded at $\mathrm{p}<0.001$ per voxel, and corrected for multiple comparisons using cluster-extent correction

662 methods through Alphasim by AFNI (58) to control family-wise error (FWE) rate at 0.05 . After identifying

663 regions from the whole-brain analysis, in which the neural representation of subjective values was

664 influenced by PTSD symptom severity, we took a leave-one-subject-out (LOSO) approach to define these 
ROIs in an un-biased way for each participant. For each left-out participant, we defined an ROI from a whole-brain analysis using data from all other participants, so this ROI definition was not influenced by the

667 left-out participant. We then sampled neural signals of the left-out participant's data from this ROI. We 668 repeated the process for all participants.

\section{Acknowledgements}

671 This research project is supported by National Center for PTSD, and NIMH Grant R21MH102634. We also

672 thank graduate student training grants from the Interdepartmental Neuroscience Program at Yale and the

673 China Scholarship Council. We thank Alicia Roy and Dr. Erin O'Brien for the recruitment and clinical

674 assessment of veterans; Dr. Michael A. Grubb for development of the task paradigm; Simon Podhajsky,

675 Yumiko Nakamura, and Pooja Salhotra for help with running experiments and data pre-processing. We also

676 thank Dr. Jutta Joormann and Dr. Hyojung Seo for suggestions and comments on data analyses.

\section{Competing interests}

679 The authors declare no financial or non-financial competing interests.

\section{References}

682 1. Harpaz-Rotem I, Tsai J, Pietrzak RH, Hoff R. The dimensional structure of posttraumatic stress symptomatology in 323,903 U.S. veterans. J Psychiatr Res [Internet]. 2014;49:31-6. Available from: https://www.ncbi.nlm.nih.gov/pubmed/24275548

2. Foa EB, Keane TM, Friedman MJ. Guidelines for treatment of PTSD. J Trauma Stress [Internet]. 2000;13(4):539-88. Available from: https://onlinelibrary.wiley.com/doi/abs/10.1023/A\%3A1007802031411

3. van der Kolk B, Greenberg M, Boyd H, Krystal J. Inescapable Shock, Neurotransmitters, and 
Addiction to Trauma: Toward a Psychobiology of Post Traumatic Stress. Biol Psychiatry

[Internet]. 1985 Mar 1 [cited 2020 Apr 28];20(3):314-25. Available from:

https://www.sciencedirect.com/science/article/pii/0006322385900617

692

4. Brown VM, Zhu L, Wang JM, Frueh BC, King-Casas B, Chiu PH. Associability-modulated loss learning is increased in posttraumatic stress disorder. Frank MJ, editor. Elife [Internet]. 2018 Jan;7:e30150. Available from: https://doi.org/10.7554/eLife.30150

5. Homan P, Levy I, Feltham E, Gordon C, Hu J, Li J, et al. Neural computations of threat in the aftermath of combat trauma. Nat Neurosci [Internet]. 2019;22(3):470-6. Available from:

6. Ruderman L, Ehrlich DB, Roy A, Pietrzak RH, Harpaz-Rotem I, Levy I. Posttraumatic Stress Symptoms and Aversion To Ambiguous Losses in Combat Veterans. Depress Anxiety [Internet]. 2016;33(7):606-13. Available from: http://www.ncbi.nlm.nih.gov/pubmed/27000639

701 7. Kable JW, Glimcher PW. The neurobiology of decision: consensus and controversy. Neuron [Internet]. 2009/09/26. 2009;63(6):733-45. Available from:

703 http://www.ncbi.nlm.nih.gov/entrez/query.fcgi? $\mathrm{cmd}=$ Retrieve \&db=PubMed\&dopt=Citation\&list_

704 uids $=19778504$

8. Gleichgerrcht E, Ibáñez A, Roca M, Torralva T, Manes F. Decision-making cognition in neurodegenerative diseases. Nat Rev Neurol [Internet]. 2010;6(11):611-23. Available from: http://dx.doi.org/10.1038/nrneurol.2010.148 Value Under Risk and Ambiguity. J Neurophysiol [Internet]. 2010;103(2):1036-47. Available from: http://jn.physiology.org/cgi/doi/10.1152/jn.00853.2009 
ambiguity in adolescence. Cogn Affect Behav Neurosci [Internet]. 2019;19(6):1364-78. Available from: https://doi.org/10.3758/s13415-019-00749-5

714 11. Kable JW, Glimcher PW. The neural correlates of subjective value during intertemporal choice. Nat Neurosci [Internet]. 2007;10(12):1625-33. Available from: http://www.ncbi.nlm.nih.gov/entrez/query.fcgi? $\mathrm{cmd}=$ Retrieve $\& \mathrm{db}=$ PubMed\&dopt=Citation\&list_ uids $=17982449$

12. Tom SM, Fox CR, Trepel C, Poldrack RA. The neural basis of loss aversion in decision-making under risk. Science (80- ) [Internet]. 2007/01/27. 2007;315(5811):515-8. Available from: http://www.ncbi.nlm.nih.gov/entrez/query.fcgi?cmd=Retrieve\&db=PubMed\&dopt=Citation\&list_

13. Fujiwara J, Tobler PN, Taira M, Iijima T, Tsutsui KI. Segregated and integrated coding of reward and punishment in the cingulate cortex. J Neurophysiol [Internet]. 2009/04/03. 2009;101(6):328493. Available from: http://www.ncbi.nlm.nih.gov/entrez/query.fcgi?cmd=Retrieve\&db=PubMed\&dopt=Citation\&list_

14. Bartra O, McGuire JT, Kable JW. The valuation system: a coordinate-based meta-analysis of BOLD fMRI experiments examining neural correlates of subjective value. Neuroimage [Internet]. salience in the human brain. Proc Natl Acad Sci U S A [Internet]. 2014;111(13):5000-5. Available from: http://www.pnas.org/lookup/doi/10.1073/pnas.1320189111 2001;4(1):95-102. Available from: 
http://www.ncbi.nlm.nih.gov/entrez/query.fcgi?cmd=Retrieve \&db=PubMed\&dopt=Citation\&list_

17. Yacubian J, Glascher J, Schroeder K, Sommer T, Braus DF, Buchel C. Dissociable systems for gain- and loss-related value predictions and errors of prediction in the human brain. J Neurosci [Internet]. 2006;26(37):9530-7. Available from: http://www.ncbi.nlm.nih.gov/entrez/query.fcgi?cmd=Retrieve\&db=PubMed\&dopt=Citation\&list_ uids $=16971537$

18. Kim SH, Yoon HS, Kim H, Hamann S. Individual differences in sensitivity to reward and punishment and neural activity during reward and avoidance learning. Soc Cogn Affect Neurosci.

19. Monosov IE, Hikosaka O. Regionally distinct processing of rewards and punishments by the primate ventromedial prefrontal cortex. J Neurosci. 2012;32(30):10318-30.

20. Fiorillo CD. Two dimensions of value: Dopamine neurons represent reward but not aversiveness. Science (80- ). 2013;341(6145):546-9.

21. Sailer U, Robinson S, Fischmeister FPS, König D, Oppenauer C, Lueger-Schuster B, et al. Altered reward processing in the nucleus accumbens and mesial prefrontal cortex of patients with posttraumatic stress disorder. Neuropsychologia. 2008;46(11):2836-44.

753 22. Elman I, Lowen S, Frederick BB, Chi W, Becerra L, Pitman RK. Functional neuroimaging of reward circuitry responsivity to monetary gains and losses in posttraumatic stress disorder. Biol Psychiatry [Internet]. 2009/07/31. 2009;66(12):1083-90. Available from:

757 23. Blake DD, Weathers FW, Nagy LM, Kaloupek DG, Gusman FD, Charney DS, et al. The development of a Clinician-Administered PTSD Scale. J Trauma Stress [Internet]. 1995;8(1):75- 
90. Available from: http://www.ncbi.nlm.nih.gov/entrez/query.fcgi?cmd=Retrieve \&db=PubMed\&dopt=Citation\&list_ uids $=7712061$

24. Paulus MP, Yu AJ. Emotion and decision-making: Affect-driven belief systems in anxiety and depression. Trends in Cognitive Sciences. 2012. making under uncertainty in obsessive-compulsive disorder. J Psychiatr Res [Internet]. 2015 Oct 1 [cited 2018 Jul 30];69:166-73. Available from: https://www.sciencedirect.com/science/article/pii/S0022395615002447?via\%3Dihub

26. Buckholtz JW, Karmarkar U, Ye S, Brennan GM, Baskin-Sommers A. Blunted Ambiguity Aversion during Cost-Benefit Decisions in Antisocial Individuals. Sci Rep [Internet]. 2017;7(1):1-

771 27. Konova AB, Lopez-Guzman S, Urmanche A, Ross S, Louie K, Rotrosen J, et al. Computational Markers of Risky Decision-making for Identification of Temporal Windows of Vulnerability to Opioid Use in a Real-world Clinical Setting. JAMA Psychiatry. 2019;08855.

28. Mukherjee D, Lee S, Kazinka R, D. Satterthwaite T, Kable JW. Multiple Facets of Value-Based Decision Making in Major Depressive Disorder. Sci Rep [Internet]. 2020;10(1):3415. Available

29. Hayes JP, Hayes SM, Mikedis AM. Quantitative meta-analysis of neural activity in posttraumatic stress disorder. Biol Mood Anxiety Disord [Internet]. 2012;2(1):1. Available from: Biology of Mood \& Anxiety Disorders 
Biobehavioral Reviews. 2015.

783

784

785

786

787

788

789

790

791

792

793

794

795

796

797

798

799

800

801

802

803

804

31. Hopper JW, Pitman RK, Su Z, Heyman GM, Lasko NB, Macklin ML, et al. Probing reward function in posttraumatic stress disorder: expectancy and satisfaction with monetary gains and losses. J Psychiatr Res [Internet]. 2007/12/11. 2008;42(10):802-7. Available from: http://www.ncbi.nlm.nih.gov/pubmed/18068725

32. May CL, Wisco BE. Reward Processing and Decision-Making in Posttraumatic Stress Disorder. Behav Ther [Internet]. 2019 Nov 26 [cited 2020 Jan 4]; Available from: https://www.sciencedirect.com/science/article/pii/S000578941930139X

33. Litt A, Plassmann H, Shiv B, Rangel A. Dissociating valuation and saliency signals during decision-making. Cereb Cortex [Internet]. 2011;21(1):95-102. Available from: http://www.ncbi.nlm.nih.gov/pubmed/20444840

34. Zhang Z, Fanning J, Ehrlich DB, Chen W, Lee D, Levy I. Distributed neural representation of saliency controlled value and category during anticipation of rewards and punishments. Nat Commun [Internet]. 2017;8(1):1907. Available from: https://doi.org/10.1038/s41467-017-02080-4

35. Shabel SJ, Wang C, Monk B, Aronson S, Malinow R. Stress transforms lateral habenula reward responses into punishment signals. Proc Natl Acad Sci U S A. 2019;116(25):12488-93.

36. Reynolds SM, Berridge KC. Emotional environments retune the valence of appetitive versus fearful functions in nucleus accumbens. Nat Neurosci. 2008;11(4):423-5.

37. Admon R, Milad MR, Hendler T. A causal model of post-traumatic stress disorder: Disentangling predisposed from acquired neural abnormalities. Trends Cogn Sci [Internet]. 2013;17(7):337-47. Available from: http://dx.doi.org/10.1016/j.tics.2013.05.005

38. Wolf RC, Herringa RJ. Prefrontal - Amygdala Dysregulation to Threat in Pediatric Posttraumatic Stress Disorder. Neuropsychopharmacology [Internet]. 2015;41(3):822-31. Available from: 
http://dx.doi.org/10.1038/npp.2015.209

806

39. Rabellino D, Densmore M, Frewen PA, Théberge J, Mckinnon C, Lanius RA. Aberrant Functional

807

808

Connectivity of the Amygdala Complexes in PTSD during Conscious and Subconscious

Processing of Trauma-Related Stimuli. PLoS One. 2016;1-12.

40. Castrellon JJ, Young JS, Dang LC, Cowan RL, Zald DH, Samanez-Larkin GR. Mesolimbic dopamine D2 receptors and neural representations of subjective value. bioRXiv Prepr. 2019;

41. Macoveanu J. Serotonergic modulation of reward and punishment: Evidence from pharmacological fMRI studies. Brain Research. 2014.

42. Gilaie-Dotan S, Tymula A, Cooper N, Kable JW, Glimcher PW, Levy I. Neuroanatomy predicts individual risk attitudes. J Neurosci [Internet]. 2014;34(37):12394-401. Available from: http:/www.ncbi.nlm.nih.gov/pubmed/25209279

43. Jung WH, Lee S, Lerman C, Kable JW. Amygdala Functional and Structural Connectivity Predicts Individual Risk Tolerance. Neuron. 2018;98(2):394-404.e4.

44. Zyphur MJ, Narayanan J, Arvey RD, Alexander GJ. The Genetics of Economic Risk Preferences. J Behav Decis Mak. 2009;22(4):367-77.

45. Chew SH, Ebstein RP, Zhong S. Ambiguity aversion and familiarity bias: Evidence from behavioral and gene association studies. J Risk Uncertain. 2012;44(1):1-18.

46. First MB, Gibbon M, Spitzer RL, Benjamin LS, Williams JBW. Structured Clinical Interview for DSM-IV® Axis II Personality Disorders SCID-II. American Psychiatric Pub; 1997.

47. Weathers FW, Litz BT, Keane TM, Palmieri PA, Marx BP, Schnurr PP. The PTSD Checklist for DSM-5 (PCL-5). Scale available from Natl Cent PTSD www ptsd va gov. 2013;10.

48. Beck AT, Steer RA, Brown GK. Manual for the Beck Depression Inventory-II. San Antonio, TX: 
Psychological Corporation; 1996.

828

829

830

831

832

833

834

835

836

49. Spielberger CD, Gorsuch RL. State-trait anxiety inventory for adults: Manual and sample: Manual, instrument and scoring guide. Consulting Psychologists Press; 1983.

50. Bernstein EM, Putnam FW. Development, reliability, and validity of a dissociation scale. 1986;

51. Keane TM, Fairbank JA, Caddell JM, Zimering RT, Taylor KL, Mora CA. Clinical evaluation of a measure to assess combat exposure. Psychol Assess A J Consult Clin Psychol. 1989;1(1):53.

52. Bernstein DP, Stein JA, Newcomb MD, Walker E, Pogge D, Ahluvalia T, et al. Development and validation of a brief screening version of the Childhood Trauma Questionnaire. Child Abuse Negl. 2003;27(2):169-90.

53. Carver CS, White TL. Behavioral-Inhibition, Behavioral Activation, and Affective Responses to Impending Reward and Punishment - the BIS/BAS Scales. J Pers Soc Psychol. 1994;67(2):319_ 33.

54. Patton JH, Stanford MS, Barratt ES. Factor structure of the Barratt impulsiveness scale. J Clin Psychol [Internet]. 1995/11/01. 1995;51(6):768-74. Available from: http://www.ncbi.nlm.nih.gov/entrez/query.fcgi?cmd=Retrieve \&db=PubMed\&dopt=Citation\&list_ uids $=8778124$

55. Blais AR, Weber EU. A Domain-Specific Risk-Taking (DOSPERT) scale for adult populations. Judgm Decis Mak J. 2006;1(1):33-47.

56. Kaufman AS. Kaufman brief intelligence test: KBIT. AGS, American Guidance Service Circle Pines, MN; 1990.

Grubb MA, Tymula A, Gilaie-Dotan S, Glimcher PW, Levy I. Neuroanatomy accounts for agerelated changes in risk preferences. Nat Commun [Internet]. 2016;7(May):1-5. Available from: http://dx.doi.org/10.1038/ncomms13822 
bioRxiv preprint doi: https://doi.org/10.1101/2020.04.14.041467; this version posted December 1, 2020. The copyright holder for this preprint

(which was not certified by peer review) is the author/funder, who has granted bioRxiv a license to display the preprint in perpetuity. It is made available under aCC-BY-NC-ND 4.0 International license.

850 58. Cox RW. AFNI: software for analysis and visualization of functional magnetic resonance neuroimages. Comput Biomed Res. 1996;29(3):162-73. 